\title{
OPTIMIZATION OF AMHS DESIGN FOR A SEMICONDUCTOR FOUNDRY FAB BY USING SIMULATION MODELING
}

\author{
Jacky Tung \\ Tina Sheen \\ Merlin Kao \\ C.H. Chen \\ Manufacturing Technology Center \\ Taiwan Semiconductor Manufacturing Company, Ltd. \\ Hsinchu, TAIWAN
}

\begin{abstract}
A $300 \mathrm{~mm}$ semiconductor fab requires a tremendous amount of investment with a great deal of process tools inside. Those process tools are connected by the automatic material handling system (AMHS), thus requiring an extremely complex AMHS network. Additionally, unlike simple products of an integrated device manufacturer (IDM) or a memory fab, foundry fab manufactures hundreds of consumer products simultaneously, subsequently creating globally an extremely high transportation volume and complex demands on the material handling system. Previously, experiential engineers designed AMHS, possibly taking months of design lead-time. However, the next generation fab AMHS design focuses not only AMHS specifications, but also operational know-how. Meanwhile, a long design lead time fails to fulfill the requirements of the fast ramp up plan. Additionally, design optimization and shrink lead time are achieved by applying simulation modeling as a design platform whenever TSMC constructs an AMHS for a new fab. Via this platform, precise simulation is performed on the AMHS specifications, fab layout, tool configuration, and process flow in-formation to ensure design success and avoid a potential AMHS bottleneck.
\end{abstract}

\section{INTRODUCTION}

A GIGAFAB $^{\mathrm{TM}}$ produces over $100 \mathrm{~K}$ wafers per month. TSMC operates three GIGAFAB ${ }^{\mathrm{TM}}$, with each one including several fully automation cluster fabs. The fully automated Fab requires AMHS to support front opening unified pod (FOUP) transportation and storage. Additionally, AMHS must provide cross-phase and cross-floor transportation to facilitate GIGAFAB ${ }^{\mathrm{TM}}$ operations.

Although experiential experts have designed AMHS for several years, scalability of GIGAFAB ${ }^{\mathrm{TM}}$ design complicates the ability to ensure design success without systematic analysis. Simulation is the most efficient means of evaluating AMHS design, estimating AMHS capability, and identifying potential bottlenecks. Actual performance does not need to be known until system release. Moreover, simulation analysis can identify the retrofit impact, provide high confidence and confirm the direction of system modification.

The rest of this paper is organized as follows: Section 2 introduces the outline of GIGAFAB ${ }^{\mathrm{TM}}$ layout. Section 3 then describes the AMHS concept. Next, Section 4 presents both AMHS simulation modeling and a case study to evaluate AMHS through simulation. Conclusions are finally drawn in Section 5, along with recommendations for future research. 


\section{OUTLINE OF GIGAFAB ${ }^{\mathrm{TM}}$ LAYOUT}

GIGAFAB $^{\mathrm{TM}}$ allows for low operation cost, a short cycle time, high ramp up agility, and excellent delivery precision. TSMC operates three $300 \mathrm{~mm}^{\text {GIGAFAB }}{ }^{\mathrm{TM}}$ currently- Fab12, Fab14, and Fab15. Each GIGAFAB ${ }^{\mathrm{TM}}$ includes several cluster fabs, and all of the cluster fabs are linked together.

The minimum capacity of one GIGAFAB ${ }^{\mathrm{TM}}$ is around $100 \mathrm{~K}$ of wafers output monthly, with thousands of process tools inside. All of the process tools are located along Intra-bay. Length of Intra-bay is around $25 \mathrm{M} \sim 50 \mathrm{M}$, including $10 \sim 30$ process tools. All of these Intra-bays are integrated through Interbay. As the main traffic route of each cluster fab, Inter-bay is connected to the neighboring fab as the cross-phase bridge. Figure 1 shows the TSMC Fab15 GIGAFAB ${ }^{\mathrm{TM}}$ with four cluster fabs.

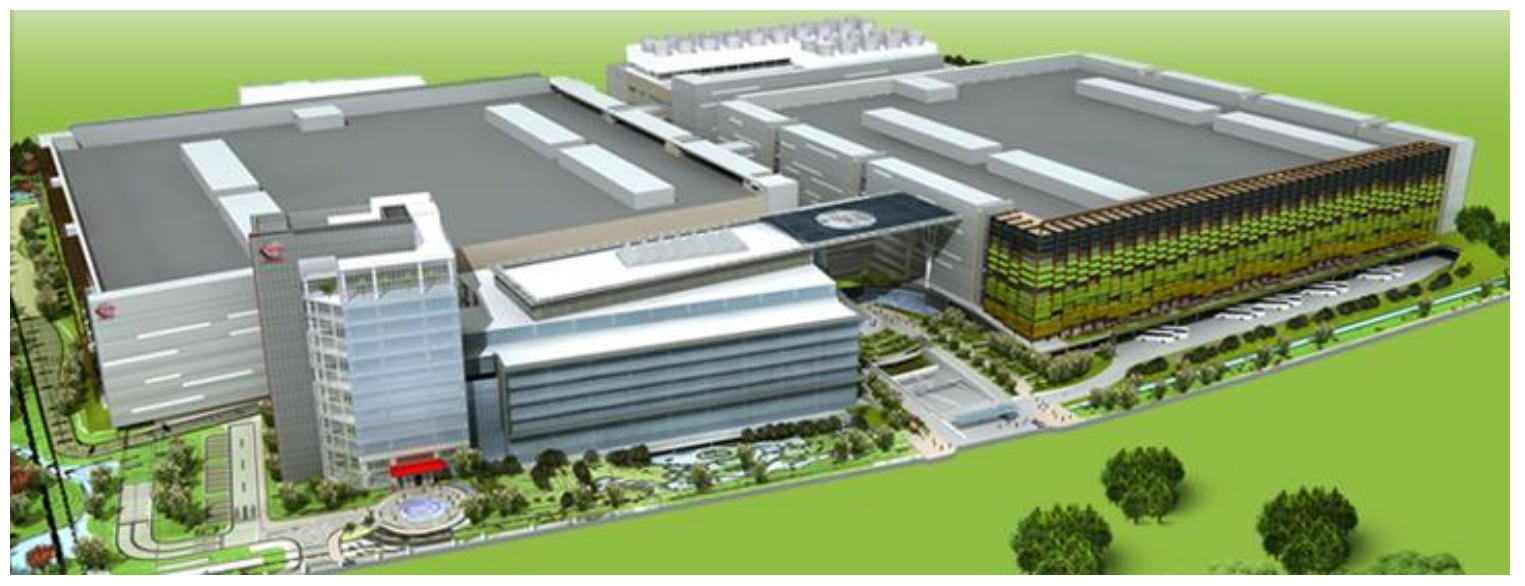

Figure 1: TSMC GIGAFAB ${ }^{\mathrm{TM}}$

\section{AMHS DESIGN}

\subsection{AMHS Devices}

AMHS has two major sub-systems: transportation system and storage devices. Figure 2 shows image of $300 \mathrm{~mm}$ fully automation fabrication with AMHS.

The major transportation system of the Integrated Circuit (IC) fabrication is over head transporter (OHT) and over head shuttle (OHS), which travels along track. The other transportation system is a conveyor. TSMC design transportation system functions as zero footprints under a ceiling to eliminate the non-production area of a fab.

Stocker and under track storage (UTS) are major storage devices. Near tool buffer (NTB) is the recently studied solution for a fast swap solution.

A special AMHS sub-system consists of cross-floor devices to support a multi-floor fab. The main cross-floor solutions are the lifter, tower stocker, or carousel.

\subsection{Design Focus}

\subsubsection{Intra-bay}

The first design factor of Intra-bay is the bay transportation requirement. Different transportation volumes require different track designs to support transport. Stocker is an additional factor. To avoid a traffic jam around stocker, the MUST item is for Intra-bay design. A shortcut is occasionally added between Intrabays to provide an alternative route for transportation. 


\subsubsection{Inter-bay}

Inter-bay is generally designed with two or four tracks to provide sufficient capability. To fulfill largescale fab transportation, a two tiered Inter-bay with six or eight tracks is implemented to provide $2 \mathrm{X}$ capability than before.

\subsubsection{Cross Phase \& Cross Floor Transportation}

Cross-phase and cross-floor transportation play the key role of fully automation GIGAFAB ${ }^{\mathrm{TM}}$ operation. Design in direct link cross-phase and cross-floor system allows for fully automatic transportation of FOUPs between fabs.

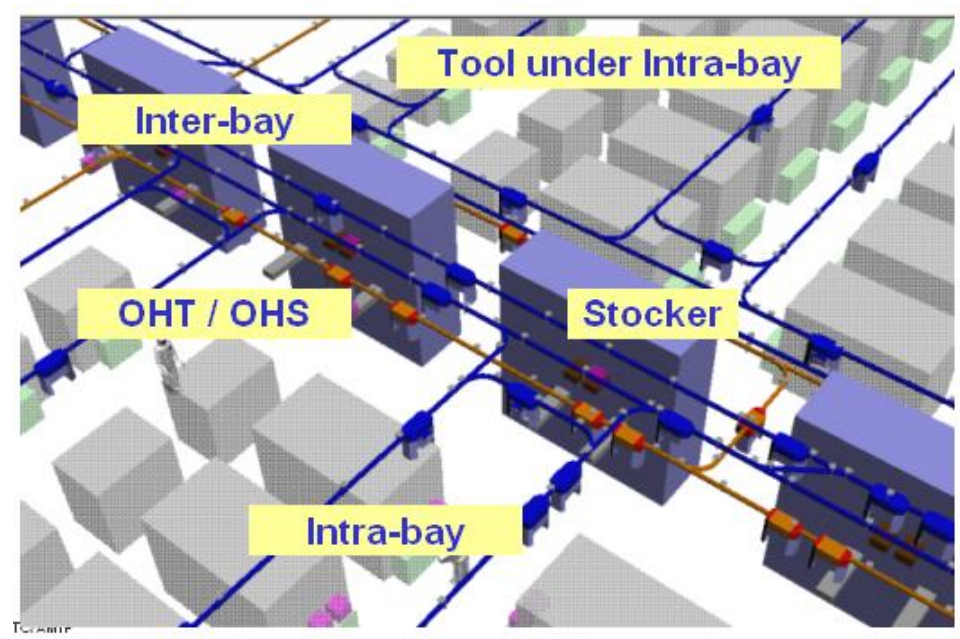

Figure 2: Sample of Fab AMHS

The experiential experts with foundry operation know-how and AMHS specification design this huge, complex, and high capability material handling system. However, it is gradually hard to guarantee and ensure first success of AMHS design because of scalability of GIGAFAB ${ }^{\mathrm{TM}}$.

\section{SIMULATION MODELING AND CASE STUDY}

The simulation model is adopted to evaluate the new fab AMHS capability, identify bottlenecks, impact of retrofit, and new device evaluation. This case study explains how to evaluate AMHS design through simulation modeling. Key performance indices (KPI) of AMHS are collected and analyzed to identify the better case.

\subsection{FAB Layout Information}

All of the cases are designed with the same tool layout. This fab layout includes around 300 process tools that belong to 100 tool types. The Fab scale is around 10 20K wafer start per month (WSPM). Figure 3 shows the Fab layout. 


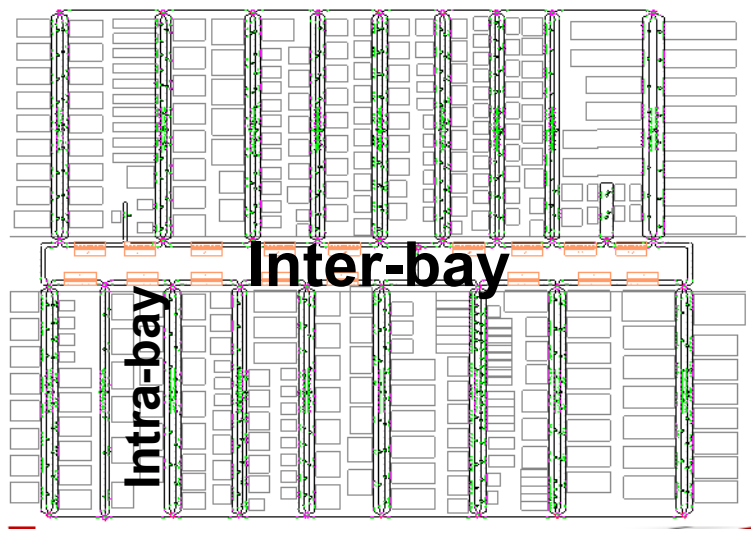

Figure 3: Fab Layout

\subsection{Tool Information}

Tool configuration is included as the effect of transportation demands. Table 1 lists the factors for tool configuration. Tool quantity refers to the same tool total quantity. LP quantity shows how many load ports are in the tool, in which the batch numbers show how many FOUPs are processed per batch. The lot processed rate shows the required processing rate in this tool. Internal buffer time and Equipment Front End Mechanism (EFEM) time indicate that the time requirement FOUP stays in a tool. MTBF shows the mean time between failures, in which MTTR shows the mean time to repair.

Table 1: Tool configuration

\begin{tabular}{|l|r|c|c|c|c|c|c|c|}
\hline Tool ID & $\begin{array}{c}\text { Tool } \\
\text { Quantity }\end{array}$ & $\begin{array}{c}\text { LP } \\
\text { Quantity }\end{array}$ & $\begin{array}{c}\text { Batch } \\
\text { Numbers }\end{array}$ & $\begin{array}{c}\text { Lot } \\
\text { Processed } \\
\text { Rate }\end{array}$ & $\begin{array}{c}\text { Internal } \\
\text { Buffer Time }\end{array}$ & EFEM time & MTBF & MTTR \\
\hline A01 & 2 & 4 & 1.00 & $100 \%$ & 0.0 & 20.0 & 43.00 & 5.31 \\
\hline A02 & 10 & 4 & 1.00 & $50 \%$ & 35.0 & 20.0 & 23.00 & 3.74 \\
\hline A03 & 2 & 4 & 1.00 & $50 \%$ & 35.0 & 20.0 & 17.00 & 2.77 \\
\hline A04 & 1 & 4 & 1.00 & $25 \%$ & 0.0 & 2.0 & 54.31 & 2.26 \\
\hline A05 & 3 & 4 & 3.00 & $100 \%$ & 21.0 & 4.0 & 30.30 & 1.59 \\
\hline A06 & 3 & 4 & 3.00 & $100 \%$ & 0.0 & 4.0 & 30.30 & 1.59 \\
\hline A07 & 2 & 4 & 6.00 & $100 \%$ & 0.0 & 4.0 & 30.30 & 1.59 \\
\hline A08 & 1 & 4 & 6.00 & $100 \%$ & 0.0 & 4.0 & 30.30 & 1.59 \\
\hline A09 & 2 & 2 & 6.00 & $100 \%$ & 0.0 & 3.0 & 55.33 & 9.76 \\
\hline A10 & 2 & 2 & 1.00 & $100 \%$ & 0.0 & 2.0 & 107.15 & 14.61 \\
\hline
\end{tabular}

\subsection{Process Flow, Merge \& Split}

Process flows are added as FOUP, which includes the "where next logic" definition. Around 50 process flows are added to this model. Each process flow includes approximately 1,000 steps. Table 2 summarizes the following process flow information: Step_Id denotes the process step; Eq id identifies the process tool in this step; Process time indicates that FOUP should be processed in a tool; and the initial lot number defines the initial condition of this step. Sometimes, it is required to sample product for measurement. Figure 4 shows the example of sampling rate when wafer is required to sample.

"Where Next" and "What's next" logic determine which FOUP should be processed. If the one of the next process tool group is available, FOUP can be delivered to this tool. If not, FOUP should go to the 
stocker or UTS for temporary storage. As for "What's next logic", the available tool selects the most feasible FOUP that is on standby in storage for processing.

FOUPs can be prioritized for processing as well. High priority FOUP is selected to process for processing first. As for same priority FOUPs, the first-in-first-out rule is implemented for selecting FOUP.

Table 2: Process flow information

\begin{tabular}{|r|l|r|r|}
\hline Step_Id & EQ_id & Process Time & Initial Lot Numbers \\
\hline 1 & A34 & 31.61 & 0 \\
\hline 2 & A27 & 297.47 & 0 \\
\hline 3 & A41 & 22.74 & 8 \\
\hline 4 & A42 & 13.33 & 0 \\
\hline 5 & A02 & 13.22 & 0 \\
\hline 6 & A67 & 3.53 & 0 \\
\hline 7 & A04 & 10.96 & 0 \\
\hline 8 & A49 & 17.01 & 0 \\
\hline 9 & A49 & 17.01 & 29.63 \\
\hline 10 & A06 & & \\
\hline
\end{tabular}

\begin{tabular}{|r|r|r|r|}
\hline 24 & $A 61$ & 2320 & $100 \%$ \\
\hline 25 & $A 75$ & 2000 & $49 \%$ \\
\hline 26 & $A 76$ & 2000 & $12 \%$ \\
\hline 27 & $A 43$ & 1657 & $100 \%$ \\
\hline
\end{tabular}

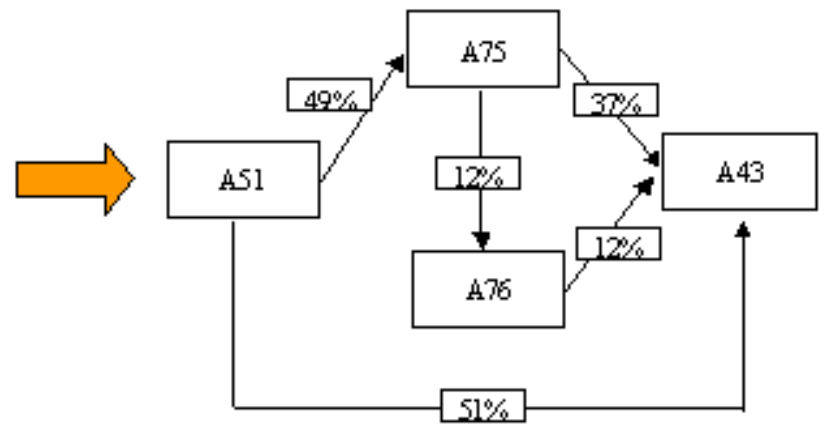

Figure 4: Sampling rate / route in a simulation model

\subsection{AMHS Concept}

Three AMHS design concepts are evaluated based on simulation:

CASE 1: Vehicle transportation system + Stocker as storage

CASE 2: Bi-directional vehicle transportation system + Carousel / UTS as storage

CASE 3: Conveyor transportation system + Stocker as storage

Table 3 lists the main system specifications and general setting.

Table 3: Case study and general setting of each system

\begin{tabular}{|c|c|c|c|c|}
\hline \multicolumn{2}{|l|}{ Case } & 1 & 2 & 3 \\
\hline \multicolumn{2}{|l|}{ Design Concept } & Vehicle+ Stocker & Vehicle+Carousel & Conveyor+Stocker \\
\hline \multirow{7}{*}{ Transportation } & Device & Vehicle + Rail & $\begin{array}{l}\text { Bi direction Vehicle } \\
\text { + Rail }\end{array}$ & Conveyor + Hoist \\
\hline & Vehicle No. & $40 \sim 100$ & $40 \sim 100$ & $\begin{array}{l}1662 \text { Conveyor + } \\
72 \text { Hoist }\end{array}$ \\
\hline & \begin{tabular}{|l} 
Speed (M/sec) \\
\end{tabular} & $3 / 1 / 0.5$ & $2 / 1 / 0.3$ & $0.8 / 0.5 / 0.2$ \\
\hline & $\begin{array}{l}\text { Acceleration } \\
\text { (M/sec2) }\end{array}$ & 0.7 & 1 & 0.5 \\
\hline & \begin{tabular}{|l}
$\begin{array}{l}\text { Hoist Time } \\
\text { (sec.) }\end{array}$ \\
\end{tabular} & $11 / 9$ & $16.5 / 11.8$ & 3 (hoist) +11(e84) \\
\hline & $\begin{array}{l}\text { MCBF or } \\
\text { Utilization }\end{array}$ & $-70 \%$ & $-70 \%$ & $200000 / 30000$ \\
\hline & MTTR(Min) & & & 30 \\
\hline \multirow{2}{*}{$\begin{array}{l}\text { Rotation } \\
\text { (Turntable) }\end{array}$} & Time (sec.) & $*$ & 9 & 11 \\
\hline & Unit & * & 38 sets & 385 sets \\
\hline \multirow{2}{*}{ Buffer } & $\begin{array}{l}\text { MCBF } \\
\text { Stocker }\end{array}$ & 38 & \begin{tabular}{|l}
3000 (MTBI) \\
10 (Carousel)
\end{tabular} & $\begin{array}{l}120,000 \\
8\end{array}$ \\
\hline & UTS & . & 1980 & $58.5 \mathrm{M}$ ( 117 sets) \\
\hline
\end{tabular}




\subsubsection{CASE 1 AMHS Design: Vehicle + Stocker}

CASE 1 design vehicle as the main transportation device. 3 Inter-bays are designed to connect Intra-bays. Central Inter-bay is designed with four tracks, and side Inter-bay is designed with two tracks. Stockers were located on each side of Intra-bay to distribute stocker to tool transportation and avoid traffic jams in center Inter-bay. Figure 5 shows the overall fab layout model.

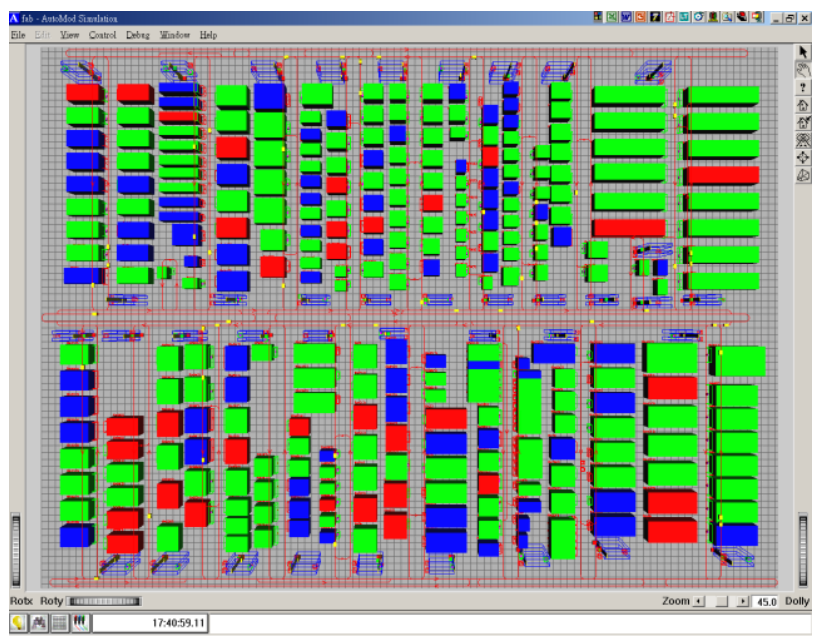

Figure 5: CASE 1 AMHS design

\subsubsection{CASE 2 AMHS Design: Bi-directional Vehicle + Carousel / UTS}

Bi-directional vehicle is designed to save traveling distance in CASE 2. Meanwhile, a turntable is the major crossing device for track design. Every Intra-bay is designed with four tracks to provide sufficient Under Track Storage (UTS). UTS are applied as main storage devices in order to save storage costs and distribute material flow, and 8 carousels are added to provide extra storage bins. Figure 6 shows the main rail design of CASE 2.

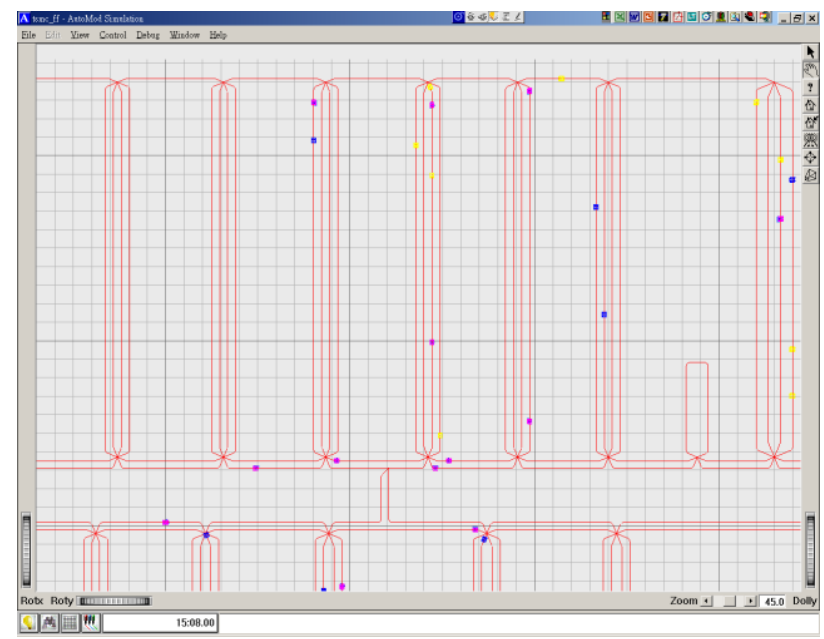

Figure 6: CASE 2 AMHS design 


\subsubsection{CASE 3 AMHS Design: Conveyor + Stocker}

The flow transport and storage system: conveyor is designed as Inter-bay for major storage and transportation. Shuttle device: Hoist sends FOUP between tool load port and conveyor as Intra-bay transportation. Few stockers are designed as additional buffer storage owing to the conveyor functioning as the main storage device. Figure 7 shows overall factory with case3 AMHS design.

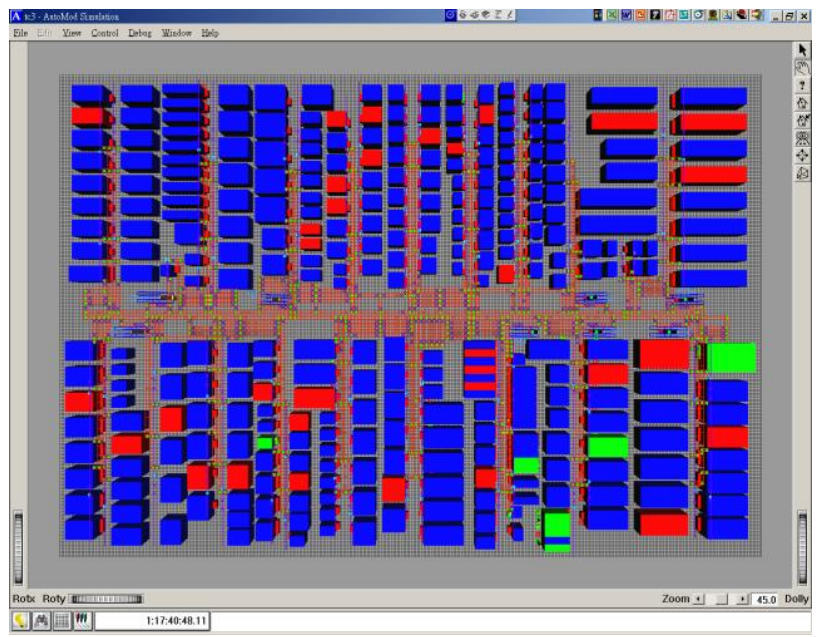

Figure 7: CASE 3 AMHS design

\subsubsection{Vehicle Control Logic}

Vehicle searching FOUP logic: The vehicle selects and picks up high priority FOUP first. FOUP with the same priority is selected by the longest wait time one.

Vehicle parking logic: The vehicle stops or parks at defined parking points. Idle vehicle can be bumped. Vehicle balance logic: The number of vehicle should be balanced in all Intra-bays. The maximum number of vehicles in one Intra-bay is constrained to 15.

Vehicle routing and deadlock prevention: When the vehicle stops at a merge point for over 30 sec., the vehicle re-routes automatically to avoid a deadlock. Figure 8 shows the vehicle with yellow color for deadlock status.

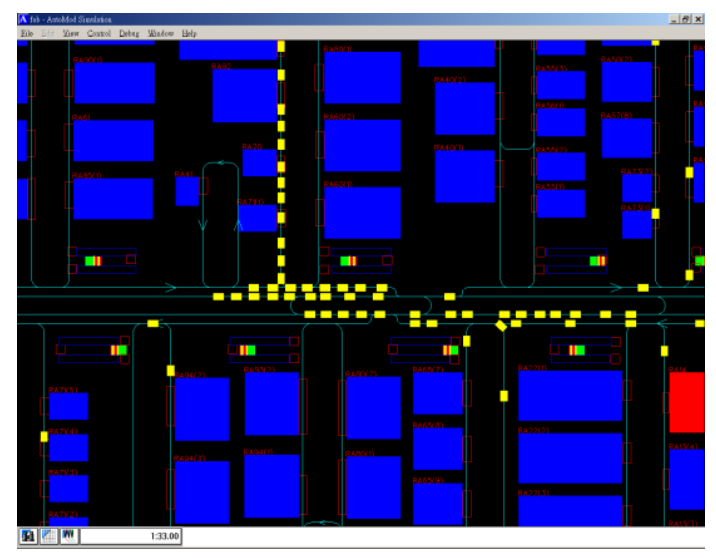

Figure 8: Vehicle dead lock 


\subsubsection{Model Development \& Running Snap}

All of three cases were developed by AutoMod ${ }^{\mathrm{TM}}$ and From-to data were generated from actual Fab transportation pattern.

OHT \& Shutter: Create by path mover system

Stocker: Create by standard module of AS/RS, ports were crested by queue or conveyor

UTS: Create by queue

Conveyor: Create by standard module of conveyor, turntables were created by customized kinematic system.

Process tools: Create by resource and queue with précised tool information.

Each run of model simulates 60 days to collect related data; the first 10 days were defined as warmup and reset without data collection.

\subsection{Performance Benchmarking}

\subsubsection{Tool Load Port Service Time}

The first index of case benchmarking is "Tool load port service time". Figure 9 summarizes the results of benchmarking, in which the total time requirement includes FOUP out + FOUP in and CASE 1 provides fast load port service time.

\section{Load Port Service Time}

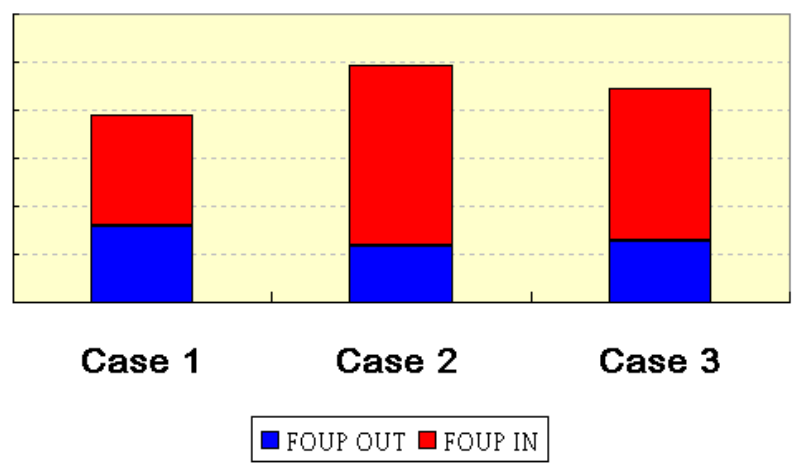

Figure 9: Benchmarking of load port service time

\subsubsection{T2T Ratio (Tool-to-Tool Ratio)}

The next index is "T2T ratio". The direct tool-to-tool transportation allows for high tool utilization, low transportation volume, and less storage requirement. Figure 10 shows final T2T ratio benchmarking, in which a high T2T ratio implies a better AMHS solution. 


\section{T2T Ratio}

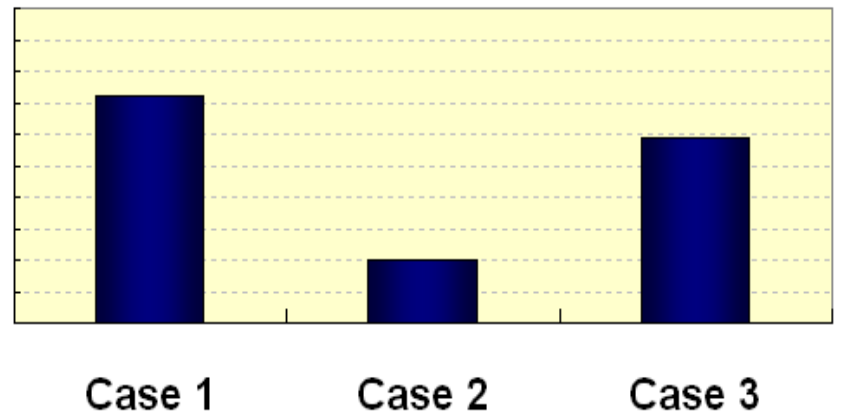

Figure 10: T2T Ratio benchmarking

\subsubsection{FOUP Cycle Time}

FOUP cycle time includes process time, idle / queue time, and transport time. Figure 11 indicates that CASE 1 provides a better AMHS service with a shorter overall cycle time.

\section{Cycle Time Benchmarking}

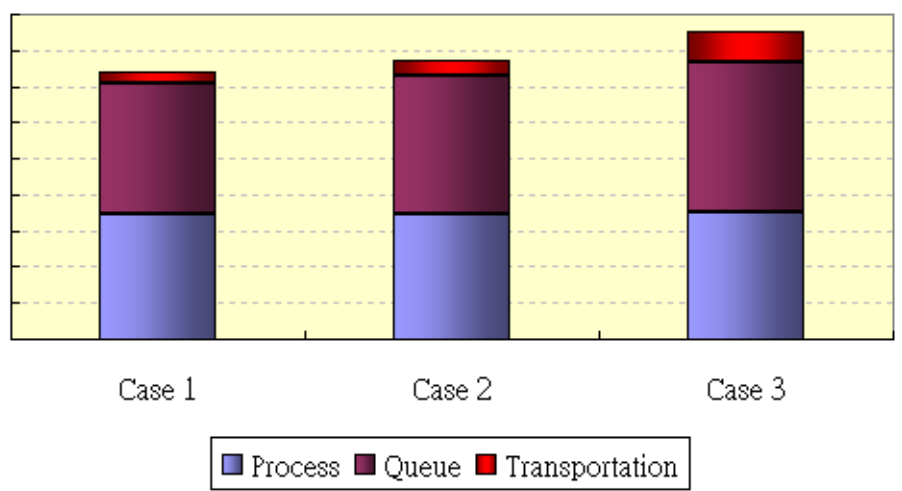

Figure 11: FOUP cycle time benchmarking

\subsubsection{FOUP Total Travel Distance}

Total travel distance refers to the usage of AMHS resource. A high traveling distance requires more transportation resources. Surprisingly, Figure 12 indicates that CASE 2 is better than CASE 1. The root cause may owing to the bi-directional traveling of vehicles. A vehicle does not require long distance onedirectional traveling. 
FOUP Total Travel Distance

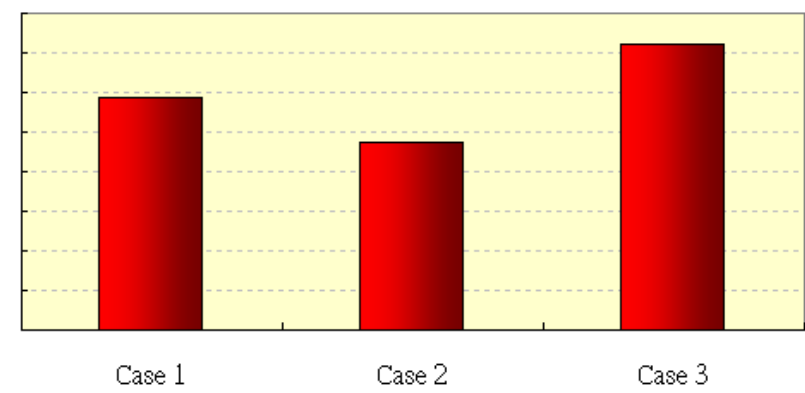

Figure 12: FOUP travel distance benchmarking

This case study demonstrates that CASE 1 performs better than all other cases. However, how to select transportation system and storage device should be depend on layout and requirements. This reflects the outcome of our current design concepts.

\section{CONCLUSIONS}

3D real time AMHS simulation requires much expertise, including fab layout, AMHS design, operational know how, and simulation skills. Despite the considerable amount of time and complexity in developing the overall simulation capability of AMHS, this extremely useful platform ensures the success for:

I. A New Fab AMHS design

II. Efficient retrofit engineering

III. New device evaluation

IV. Performance tuning and optimization

V. Vender selection

TSMC has developed its simulation capability for several years. The simulation platform facilitates decision-making involving GIGAFAB ${ }^{\mathrm{TM}}$ AMHS design and operation.

\section{REFERENCE}

Jimenez, Wright, Grosser, Bell, Adikaram, and Davila. 2010. AMHS factors enabling small wafer lot manufacturing in semiconductor wafer fabs. In 2010 Winter Simulation Conference.

Schulz. M., Stanley T.D., Renelt B., Sturm R. 2000. Simulation based decision support for future 300mm automated material handling. In 2000 Winter Simulation Conference.

Ying Wang, Chen Zhou. Fluid-based simulation approach for high volume conveyor transportation systems. 2004. Journal of Systems Science and Systems Engineering September 2004, Volume 13, Issue 3, pp 297-317

\section{AUTHOR BIOGRAPHIES}

Jacky Tung is a manger in Manufacturing Technical Center, TSMC. He is in charged of new Fab AMHS design and simulation evaluation. During his 18 years experience in material handling system, Jacky de- 
signs most of TSMC GIGAFAB ${ }^{\mathrm{TM}}$ AMHS and evaluate it through simulation. He has a master's degree in naval architecture engineering from National Taiwan University. His email address is cftung@tsmc.com

Tina Sheen is a deputy director in Manufacturing Technology Center, TSMC. During her 22 years experience in semiconductor industries, she held various manger positions in manufacturing. She is TSMC assignee of ISMI (International SEMATECH Manufacturing Initiative) $450 \mathrm{~mm}$ Program Advisory Group (450 PAG), Manufacturing Technology Program Advisory Group (MTP PAG) \& ISSM, International Symposium on Semiconductor Manufacturing, International Program Committee. She won the Woman Innovated Award from the president of Taiwan in 2012. Her email address is syshen@tsmc.com

Merlin Kao is a technical manager in Manufacturing Technology Center, TSMC. He has 18 working experience in semiconductor industries and manger position for manufacturing \& AMHS performance evaluation. He obtained MBA in Industrial Management at National Cheng Kung University, Taiwan. His email address ismlkao@tsmc.com

C.H. Chen is a director of Taiwan Semiconductor Manufacturing Company's Manufacturing Technology Center. He joined TSMC in 1989 and has held multiple positions during his 24 years of career in TSMC. He was a Fab leader with over one decade's experience and was appointed as a general manager of TSMC joint venture Fab, SSMC in Singapore in 2003 and TSMC China in 2009. He graduated from National Chiao Tung University with a master degree in photonics. 